\title{
On the ways of forming information structures for modeling objects, environments and processes
}

\author{
N.V. Zenyutkin' ${ }^{1}$, D.I. Kovalev³, ${ }^{2,}$ E.V. Tuev ${ }^{4}$, E.V. Tueva ${ }^{4}$ \\ ${ }^{1}$ Siberian Federal University, 79, Svobodny pr., Krasnoyarsk, 660041, Russia \\ ${ }^{2}$ Krasnoyarsk State Agrarian University, 90, Mira pr., Krasnoyarsk, 660049, Russia \\ ${ }^{3}$ Krasnoyarsk Science and Technology City Hall of the Russian Union of Scientific and \\ Engineering Associations, 61, Uritskogo street, Krasnoyarsk, 660049, Russia \\ ${ }^{4}$ Reshetnev Siberian State University of Science and Technology, 31, Krasnoyarsky Rabochy \\ Av., Krasnoyarsk, 660037, Russia \\ * E-mail: grimm7jow@gmail.com
}

\begin{abstract}
The paper discusses the well-known concepts of matter, objects, environments, information and information field in relation to the proposed information representation of the interaction of objects and environments based on the description of their information structures. An overview of the methods of forming information structures for modeling objects, environments and processes is given. It is shown that without using the concept of the environment, as an entity in which objects exist and are reflected in time and in a certain space, it is not always possible to accurately describe the change in the state of an object. This is due to the fact that the current state of an object depends both on the action of other objects on it and on its ability to reflect a specific physical or other environment in which it and other objects exist. From the presented review of approaches to object-oriented methods of modeling objects, environments and processes, it can be concluded that it is fundamentally important to take into account the physical and spatial environment in which they interact in object methods of modeling and programming complex objects and systems. Using programming languages, we can create and even manage the information structures of objects and environments. They are called abstract because they are not informational representations of the corresponding physical objects and environments. An example of such capabilities are special programming languages for creating virtual objects and virtual worlds. Created on the basis of special programming languages, virtual simulators help specialists of various professions acquire skills in managing real objects, technological processes, etc.
\end{abstract}

Keywords: information structure, object, environment, modeling, programming 
УДК 658.512 .001 .56

\title{
О способах формирования информационных структур для моделирования объектов, сред и процессов
}

\author{
Н.В. Зенюткин ${ }^{1}$, Д.И. Ковалев ${ }^{2,3^{*}}$, Е.В. Туев ${ }^{4}$, Е.В. Туева ${ }^{4}$ \\ ${ }^{1}$ Сибирский федеральный университет, пр. Свободный, 79, Красноярск, \\ 660041, Россия \\ ${ }^{2}$ Красноярский государственный аграрный университет, пр. Мира, 90, \\ Красноярск, 660049, Россия \\ ${ }^{3}$ Красноярский краевой Дом науки и техники Российского Союза научных и \\ инженерных общественных объединений, ул. Урицкого, 61, Красноярск, \\ 660049, Россия \\ ${ }^{4}$ Сибирский государственный университет науки и технологий имени \\ академика М.Ф. Решетнева, пр. Красноярский рабочий, 31, Красноярск, 660037, \\ Россия \\ * E-mail: grimm7jow@gmail.com
}

\begin{abstract}
Абстракт. В работе рассматриваются известные понятия материи, объектов, сред, информации и информационного поля применительно к предлагаемому информационному представлению взаимодействия объектов и сред на основе описания их информационных структур. Дается обзор способов формирования информационных структур для моделирования объектов, сред и процессов. Показано, что без использования понятия среды, как сущности, в которой во времени и в определенном пространстве существуют и отражаются объекты, не всегда достаточно точно можно описать изменение состояния объекта. Это связано с тем, что текущее состояние объекта зависит, как от действия на него других объектов, так и от его способности отражать конкретную физическую или другую среду, в которой он и другие объекты существуют. Из представленного обзора подходов к объектно-ориентированным методам моделирования объектов, сред и процессов можно сделать вывод, что принципиально важно в объектных методах моделирования и программирования сложных объектов и систем учитывать физическую и пространственную среду, в которой они взаимодействуют. Используя языки программирования, мы можем создавать и даже управлять информационными структурами объектов и сред. Их называют абстрактными, так как они не являются информационными представлениями соответствующих физических объектов и сред. Примером таких возможностей являются специальные языки программирования для создания виртуальных объектов и виртуальных миров. Созданные на основе специальных языков программирования виртуальные тренажеры помогают специалистам различных профессий получать навыки управления реальными объектами, технологическими процессами и т.д.
\end{abstract}

Ключевые слова: информационная структура, объект, среда, моделирование, программирование 


\section{1. Введение}

При решении задач управления различными объектами возникает необходимость идентификации объектов на основе оценок эквивалентности и классификации их информационных образов или соответствующих информационных представлений [1-4]. Математические модели также являются информационными, так как математическими символами или математическим языком описываются свойства и признаки объектов, которые отражают их соответствующую сущность. Если исследователь (интеллектуальная структура) понимает смысл, заключенный в информационных представлениях объектов, которые могут выражаться в любой информационной форме (изображение, звук и т.д.), то это означает, что он может распознавать, классифицировать и управлять ими.

Распознавание информационных образов можно отнести к задачам теории информации, которые связаны с теоретическими и практическими основами построения адаптивных систем управления, систем управления знаниями, искусственного интеллекта, систем принятия решений и т.д.

Результат принятия решений экспертами или системами с искусственным интеллектом часто определяется адекватностью представления объекта соответствующей моделью, а также результатами определения эквивалентности или классификации объектов на основе анализа их информационных образов.

Современная теория распознавания образов является разделом науки, который находится на этапе накопления знаний об информационных представлениях объектов и их взаимосвязей.

В данной работе не будут рассматриваться конкретные методы распознавания, их точность и т.д. Нас интересуют наиболее общие и основополагающие проблемы распознавания:

- какие информационные признаки объектов являются наиболее общими для их классификации;

- какими информационными признаками должны обладать эквивалентные объекты;

- всегда ли эквивалентные информационные образы принадлежат эквивалентным объектам;

- могут ли эквивалентные объекты иметь неэквивалентные информационные образы;

- $\quad$ на основе, какой информации об объектах задача определения их эквивалентности теоретически имеет решение. 
Для ответов на эти вопросы в работах [5-10] введены понятия информационной структуры объекта и его окружающей среды, которые базируются на философских представлениях об «отражении» и «информации».

В работе [11] предложено информационные структуры классифицировать, как открытые, закрытые и смешанные в соответствие с принадлежностью объектов к открытым, закрытым и смешанным (связанным) средам отражения. Это дает возможность рассматривать информационные представления объектов и сред отражения в пространствах их совместного существования, что важно для моделирования и идентификации сложных пространственных объектов и систем.

Решение задачи распознавания объектов рассматривается, как интеллектуальная, которая непосредственно связана с получением знаний через отражение информационных представлений об объектах в интеллектуальной среде.

Определение эквивалентности и классификации объектов основывается на анализе взаимосвязей их информационных структур, которые определяются на основе теории множеств. Такой подход дает возможность описывать, анализировать и делать выводы об эквивалентности и классификации объектов в виде, который удобен для обсуждаемых проблем распознавания [12-15].

В работе рассматриваются известные понятия материи, объектов, сред, информации и информационного поля применительно к предлагаемому информационному представлению взаимодействия объектов и сред на основе описания их информационных структур. Соответственно определены следующие понятия.

Информационная структура связывает информационные представления результатов взаимодействия или отражения объектов и сред с информационными представлениями их способностей отражения и информационными представлениями отраженных объектов и сред.

Информационный образ есть информационное представление результатов отражения объекта или среды при их взаимодействии.

Функции отражения являются информационным представлением способности отражения объекта или среды.

Информация об отраженных объектах и средах есть их информационное представление, которое в наиболее полном виде характеризуется их информационной структурой.

Полная совокупность информационных представлений отражений объектов и сред определяет их общее информационное поле. 


\section{2. Основные понятия об информационных структурах}

Любая задача управления предполагает наличие объекта управления. Объектами управления могут быть различные физические и не физические тела как живой, так и не живой природы.

Известно [1,2], что теория управления оперирует с моделями объектов, которые могут быть математическими, физическими, логическими и т. д.

Реальное управление возможно только реальными объектами.

Поэтому при решении задачи теоретического синтеза управления реальным объектом важно достоверное или адекватное описание его модели. В противном случае решение теоретической задачи управления останется только теорией, которую не возможно перенести на практику.

Для достоверного описания модели объекта используются различные методы и алгоритмы идентификации.

Под идентификацией [2] в широком смысле понимается получение или уточнение модели в тех или иных терминах по экспериментальным данным, полученным при исследовании реального объекта.

Таким образом, экспериментальные данные являются мерой или оценкой модели объекта. Однако необходимо заметить, что по экспериментальным данным мы также оцениваем или представляем реальный объект в виде информационных образов.

Следовательно, экспериментальные данные или информация об объекте и есть в нашем понимании реальный объект для задачи построения его модели.

Поэтому перед исследователями всегда стоит задача информационного описания объекта на основе использования различных оценивающих приборов и датчиков, к которым также можно отнести органы чувств и интеллектуальные способности самого исследователя.

Исследователь может представить информационное описание объекта в виде его информационной структуры. Современные компьютерные технологии предоставляют возможность обрабатывать и сохранять результаты исследований в виде соответствующей информации в базах данных компьютеров, которые могут быть объединены в различные информационные сети.

Перед каждым исследователем встает вопрос, что такое полное описание объекта или что собой представляет полная информационная структура объекта и чем она отличается от реального объекта?

Объяснение этого вопроса непосредственно связано с философскими понятиями материи, информации, существования материи и информации. 
Известно большое разнообразие этих философских понятий, например [3-5]. В работе [13] представлен обзор употребляемых понятий термина информации, который показывает, что феномен информации имеет огромное значение для понимания законов построения и развития Вселенной. На наш взгляд, наиболее общая взаимосвязь между материей (объектами) и информацией представлена в работах, которые можно отнести к теоретическим представлениям об информационном поле (ИП) [6-8] и основополагающим принципам построения интеллектуальных систем [11].

В соответствие с [7] под ИП объекта необходимо понимать «поле отражения объекта в окружающей среде, структура которой соответственно видоизменяется». Связь между материей и информацией в [7] характеризуется следующим образом. «Неоднородность пространственного распределения материи есть ее структура, а ее существование во времени есть движение материи. Структура (или материя) в движении (изменение) есть информация, без которой материя не может существовать, как не может существовать и информация без материи. Следовательно, информация есть философская категория, равнообъемная категории материи и парная ей».

В работе Полякова А.О. и Лачинова В.М. по информодинамике [11] отмечена аналогичная связь между материей и информацией: “неразделимость физической и информационной половинок Мира. Но эта неразделимость обязательна только для Реального Мира, может существовать и множество чисто виртуальных Миров”.

Отметим, что виртуальные миры могут создаваться и существовать только в физическом носителе информации (например, человек или компьютер), а это соответствует неразделимости физической и информационной составляющей Мира. Можно предположить, что авторы [11] выделяют виртуальный Мир в самостоятельный, так как он не имеет соответственного физического аналога. Однако это не означает, что виртуальный Мир является самостоятельно рожденной и развивающейся информационной сущностью. Для его рождения необходим физический субъект.

Таким образом, информационное представление изменения объектов во времени и есть ИП объектов [7], которое интегрально состоит из информационных полей каждого объекта и изменяется в соответствии с изменениями информационных полей этих объектов.

Н. Винер [5] отмечает, что «информация есть информация, а не материя и не энергия». Это высказывание согласуется с определениями информации, которые приведены в [7,11].

Колмогоров А.Н. и Глушков В.М. [6,7] под понятием информации понимали характеристики внутренней организованности материальной системы, которые проявляются во множестве ее состояний. 
Под термином информационная структура объекта или среды часто понимают базы данных их информационных признаков [16-18].

Понятие информационной структуры объекта, как его определенной информационной сущности, должно включать не только информационное представление результата изменения объекта во времени, но и информационные представления способности к этому изменению, что соответствует внутренней организованности объекта [13], а также информационное представление причин, которые привели к результату изменения [4]. Тоже относится к понятию информационной структуры среды.

Следовательно, понятие модели объекта и его окружающей среды должно означать более или менее точное представление их информационных структур в нашем сознании или понимании.

\section{3. Терминология и основные определения}

В соответствие с [7,11-13] можно ввести следующее определение понятия информационной структуры окружающей среды объектов или среды. При этом под окружающей средой в широком смысле понимаем физическую или любую другую сущность, в которой во времени и в определенном пространстве существуют, отражают и отражаются объекты. Например, воздушная, морская, социальная, интеллектуальная, операционная среды и т.д.

\section{Определение 1.1}

Под термином информационная структура среды будем понимать информационную сущность, которая характеризует связь информационного образа этой среды с ее функциями отражения и информацией об объекте отражения.

Объясним термины, используемые в этом определении.

Информационный образ среды есть информационное представление изменения среды в результате взаимодействия или отражении в ней объектов. В соответствие с определением 1.1 информационный образ среды формируют ее функции отражения, а также информация об объектах отражения.

Функции отражения являются информационным представлением способности среды отражать объекты, а информация об объекте отражения есть информационное представление объекта, которое определяет причину изменения информационного образа среды. Если нет объекта, то нет его отражения в среде. Способность среды отражать объекты является ее индивидуальной внутренней характеристикой, которая может использоваться для определения отличия и сходства между средами. Эту характеристику можно назвать 
индивидуальным паспортом среды, который формирует ее состояние или изменение в зависимости от отраженных объектов. Поэтому по информационным представлениям способности среды отражать объекты можно идентифицировать и классифицировать среды. Информация об отраженном объекте есть его информационное представление. В наиболее полном виде информационное представление отраженного объекта характеризуется его информационной структурой.

Следовательно, информационный образ среды, как информационное представление результата отражения в ней объектов, содержит в себе всю информацию об отраженных объектах и способностях среды отражать эти объекты.

Соответственно можно сформулировать следующие определения информационного образа среды, ее функций отражения и информации об объекте отражения.

Определение 1.2

Под информационным образом среды будем понимать информационное представление результата изменения этой среды при отражении в ней объекта.

Определение 1.3

Под функциями среды отражения будем понимать информационное представление ее способностей отражать объекты.

Определение 1.4

Под информацией об объекте отражения будем понимать его информационное представление.

В соответствие с определением 1.1 информационную структуру среды отражения в наиболее общем виде можно описать, как

$$
\mathrm{I}_{\mathrm{eo}}=\mathrm{f}_{\mathrm{eo}}\left(\mathrm{I}_{\mathrm{o}}\right)
$$

где $\mathrm{I}_{\mathrm{eo}}$ - информационный образ среды отражения, $\mathrm{f}_{\mathrm{eo}}$ - функции среды отражения, $\mathrm{I}_{\mathrm{o}}$ информация об объекте отражения.

Необходимо отметить, что выражение (1) только формально характеризует, что существует связь между информационным образом среды с ее функциями отражения и информацией об объекте отражения. Качественные и количественные оценки этой связи возможны только при анализе информационных представлений конкретных сред. Далее будет показано, что аналогично существует связь информационного образа объекта с его функциями отражения и информацией об отраженной среде. Эта связь объясняется тем, что в результате взаимодействия объекта и среды всегда возникают соответствующие изменения состояния, как объекта, так и среды, которые также проявляются в их информационных структурах. 
В соответствие с [7,11-13] введем определение информационной структуры объекта. При этом под объектом в широком смысле понимаем физическую или любую другую сущность, которая существует во времени и в определенном пространстве окружающей среды.

\section{Определение 1.5}

Под термином информационная структура объекта будем понимать информационную сущность, которая характеризует связь информационного образа объекта с его функциями отражения и информацией об отражаемой окружающей среде.

Информационный образ объекта следует понимать, как информационное представление изменения объекта в результате его взаимодействия с окружающей средой. В соответствие с определением 1.5 информационный образ объекта формируют его функции отражения, а также информация об отражаемой окружающей среде. При этом окружающая среда может включать в себя и другие объекты. Функции отражения являются информационным представлением способности объекта отражать среду. Если нет среды, то объект не может ее отразить. Способность объекта отражать среду является его индивидуальной внутренней характеристикой, которая может использоваться для определения отличия и сходства между объектами. Поэтому по информационным представлениям способности объекта отражать среду можно идентифицировать и классифицировать объекты. Информация об отражаемой среде определяет причину, которая вызвала изменения информационного образа объекта, так как способность объекта отражать среду проявляется только тогда, когда объект попадает в соответствующую среду. Информацию об отражаемой среде необходимо понимать, как информационное представление этой среды. В наиболее полном виде информационное представление среды есть ее информационная структура.

Следовательно, информационный образ объекта содержит в себе всю информацию об отраженных средах и индивидуальных внутренних способностях объекта отражать эти среды.

Соответственно можно сформулировать следующие определения информационного образа объекта, его функций отражения и информации об отражаемой среде.

Определение 1.6

Под термином информационный образ объекта будем понимать информационное представление изменения объекта в результате его отражения окружающей среды.

\section{Определение 1.7}

Под функциями отражения будем понимать информационное представление способностей объекта отражать среду. 
Определение 1.8

Под информацией об отражаемой среде будем понимать информационное представление этой среды.

В соответствие с определением 1.5 информационную структуру объекта можно описать, как

$$
I_{o e}=f_{o e}\left(I_{e}\right)
$$

где $\mathrm{I}_{\mathrm{oe}}$ - информационный образ объекта, $\mathrm{f}_{\text {ое }}$ - функции отражения объекта, $\mathrm{I}_{\mathrm{e}}$ - информация об отражаемой среде.

При этом понимаем, что отражение объектов и сред происходит во времени и в определенном пространстве. Поэтому их информационные структуры также характеризуют эти отражения во времени и в пространстве.

Покажем на абстрактном примере, какой смысл заключен в термине информационная структура.

Предположим, что имеются датчики информации, которые теоретически точно и полно фиксируют, но не влияют на результат отражения, способность отражения, отражаемые среды и объекты. Поэтому, полученная информация только характеризует отражение объектов и сред, а не является их физической сущностью.

Зафиксированные результаты изменения состояния сред и объектов при отражении

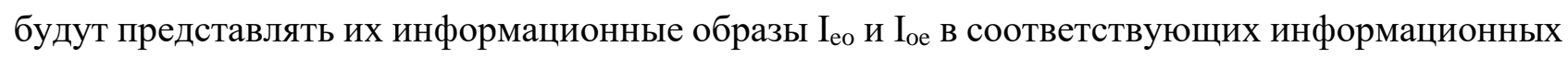
структурах сред и объектов.

Зафиксированные способности сред и объектов изменять свои состояния при отражении, представляют их функции отражения $f_{\text {eо }}$ и $f_{\text {oe }}$ в соответствующих информационных структурах сред и объектов.

Зафиксированные отраженные среды и объекты будут представлять о них информацию Іо и $\mathrm{I}_{\mathrm{e}}$ в соответствующих информационных структурах сред и объектов.

Этот пример показывает, что термин информационная структура означает только информационное представление отражения объектов и сред, но не их физическое взаимодействие. С помощью информационных структур мы можем представить и даже продемонстрировать на экране компьютера, что в процессе сжигания топлива выделяется тепло. Но это не означает, что мы в компьютере реально сжигаем топливо. Информационные структуры нам дают возможность моделировать объекты, среды и их процессы взаимодействия, а также демонстрировать результаты этих взаимодействий. 
Необходимо понимать, что восприятие окружающего мира человеком, животным или насекомым различно и зависит от их органов чувств, интеллектуальных способностей и т. д. Поэтому информационные представления отражения одних и тех же объектов и сред для них будут различны. Однако это не означает, что информационных структур не существует и их нельзя изучать, а результаты использовать для развития информационных технологий.

В соответствие с тем, что материя или объекты существуют в движении или изменении, а информационная составляющая Мира неразделима с физической, то с нашей точки зрения, выражения (1), (2) описывают основной закон изменения ИП. Он характеризует информационное представление изменчивости объектов и сред.

\section{Определение 1.9}

Под основным законом изменения информационного поля будем понимать изменение информационных образов объектов и сред в соответствие с их функциями отражения и информацией об отраженных средах и объектах.

На практике для описания и исследования информационных структур могут использоваться различные языки программирования (в частности, объектноориентированные).

В объектно-ориентированном программировании и моделировании [3] есть понятие объект, но нет специально определенного понятия среда. Можно предположить, что в этих языках под средой отражения конкретного объекта следует понимать совокупность других объектов, которые могут влиять на изменение его состояния. Важно, что без использования понятия среды, как сущности, в которой во времени и в определенном пространстве существуют и отражаются объекты, не всегда достаточно точно можно описать изменение состояния объекта. Это связано с тем, что текущее состояние объекта зависит, как от действия на него других объектов, так и от его способности отражать конкретную физическую или другую среду, в которой он и другие объекты существуют. Например, информационная модель морского судна, которое находится в водной среде, должна существенно измениться, если судно находится на суше. В этом случае взаимодействие судна с другими объектами, например с экипажем, который запустил двигатель, не дает ему возможность передвигаться по суше. Таким образом, среда, в которой находится судно, является существенным фактором для его движения, но не влияет на запуск двигателя. Этот пример показывает, что для судна окружающей средой являются, как объекты, так и физическая среда (вода, суша, тепловое окружение и т.д.), которые могут влиять на его текущее состояние. 


\section{4. Заключение}

Из представленного обзора подходов к объектно-ориентированным методам моделирования объектов, сред и процессов можно сделать вывод, что принципиально важно в объектных методах моделирования и программирования сложных объектов и систем учитывать физическую и пространственную среду, в которой они взаимодействуют.

Заметим, что, используя языки программирования, мы можем создавать и даже управлять информационными структурами объектов и сред, которые существуют или существовали только абстрактно. Их можно назвать абстрактными, так как они не являются информационными представлениями соответствующих физических объектов и сред. Примером таких возможностей являются специальные языки программирования для создания виртуальных объектов и виртуальных миров. Созданные на их основе тренажеры, помогают специалистам различных профессий получать навыки управления реальными объектами, технологическими процессами и т.д. Однако виртуальные объекты и среды могут быть основой для создания реальных объектов. Используя специальные среды компьютерного проектирования CAD/CAM, инженеры разрабатывают виртуальные объекты, которые далее используются для производства реальных изделий. В результате эти виртуальные объекты становятся соответствующими информационными представлениями физически существующих станков, кораблей и т.д. Следовательно, интеллектуальные способности человека дают возможность получать знания из информации и на этой основе создавать новую информацию, а также новые объекты и среды, которых ранее не было в природе.

\section{Список литературы}

[1] Корн, Г. Справочник по математике / Г. Корн, Т. Корн. - 4-е изд. - М.: Наука, 1978. - 719 c.

[2] Алгазинов, Э. К. Анализ и компьютерное моделирование информационных процессов и систем / Э. К. Алгазинов, А. А. Сирота; под общ. ред. д-ра техн. наук А. А. Сироты. М.: Диалог-МИФИ, 2009. - 416 с.

[3] Горлушкина, Н. Н. Системный анализ и моделирование информационных процессов и систем / Н. Н. Горлушкина. - СПб: Университет ИТМО, 2016. - 120 с.

[4] Шенон, К. Работы по теории информации в кибернетике / К. Шенон. - М.: ИЛ, 1963.

[5] Винер, Н. Кибернетика, или Управление и связь в животном и машине / Н. Винер; пер. с англ. И. В. Соловьева, Г. Н. Поварова; под ред. Г. Н. Поварова. - 2-е издание. - М.: Наука; Главная редакция изданий для зарубежных стран, 1983. - 344 с.

[6] Глушков, В. М. Мышление и кибернетика / В. М. Глушков // Вопросы философии. - 1963. 
- № 1. - C. 10-24.

[7] Колмогоров, А. Н. Три подхода к определению понятия «количество информации» / А. Н. Колмогоров // Проблемы передачи информации. - 1965. - Т.1. - Вып.1. - С. 2538.

[8] Блюменау, Д. И. Информация и информационный сервис. Л.: Наука, 1989.

[9] Попов, Э. В. Экспертные системы решения неформализованных задач в диалоге с ЭВМ / Э. В. Попов. - М.: Наука, 1987.

[10] Поспелов, Д.А. Данные и знания. Представление знаний / Поспелов Д. А. // Искусственный интеллект. Кн. 2: Модели и методы: Справочник / под ред. Д. А. Поспелова. - М.: Радио и связь, 1990. 7-13 с.

[11] Лачинов, В. М. Информодинамика / В. М. Лачинов, А. О. Поляков - СПб, издательство СПбГТУ, 1999.

[12] Кузнецов, Н. А. Состояние, перспективы и проблемы развития информатики / Н. А. Кузнецов, Р. И. Полонников, Р. М. Юсупов В кн. Теоретические основы и прикладные задачи интеллектуальных информационных технологий. - СПб, Изд. СПИИРАН, 1998.

[13] Вебер, А. В. Knowledge-технологии в консалтинге и управлении предприятием / А. В. Вебер , А. Д. Данилов, С. И. Шифрин. - СПб: Наука и Техника, 2003.

[14] Шапошников, Г. Г. Классификация информационных систем, используемых в предпринимательской деятельности / Г. Г. Шапошников // Вопросы современной юриспруденции. - 2016. - №. 5(56). - С. 70-79.

[15] Мищенко, О. В. Классификация событий для системы анализа надёжности функционирования технических средств аэронавигационной системы России / О. В. Мищенко, А. А. Апанасов, В. И. Семенов // Научный вестник МГТУ ГА. - 2012. - № 180. - С. 60-64.

[16] Конюхов, В. Г. База данных. Понятие, значение и роль в современном мире / В. Г. Конюхов // Системные технологии. - 2017. - № 24. - С. 61-63.

[17] Васильева, К. Н. Реляционные базы данных / К. Н. Васильева, Г. Я. Хусаинова // Colloquium-journal. - 2020. - №2(54). - C. 22-23. doi:10.24411/2520-6990-2020-11217.

[18] Зыкин, С. В. Технология раздельного формирования многомерных данных / С.В. Зыкин, С. В. Мосин, А. Н. Полуянов // Advanced Engineering Research. - 2016. - 2(85). - С. 121-128. 